\title{
Quality check of iron chelates applied to ornamental shrubs on sphagnum peat
}

\author{
By B. van LUIT and R. BOXMA \\ Institute for Soil Fertility, Haren (Gr.), The Netherlands
}

\begin{abstract}
SUMMARY
Responses of Chamaecyparis lawsoniana 'Alumii' and Chaenomeles superba 'Nicoline' on moss peat (slightly decomposed young Sphagnum) to various added iron chelates and lime were studied in pot experiments. The best results were obtained with $\mathrm{Fe}$-EDDHA, which was a more effective Fe source at $\mathrm{pH} 5.6$ and 6.3 than either Fe-EDTA or Fe-DTPA for Chaenomeles. Iron deficiency symptoms were mild at $\mathrm{pH} 4.5$ and differences in effectiveness between chelates could not be established at that $\mathrm{pH}$. Results obtained with another commercial iron chelate, Fe-EDDHA, containing only one third of its $\mathrm{Fe}$ as Fe-EDDHA, were inferior. The remaining $\mathrm{Fe}$ fraction proved largely ineffective in controlling foliar chlorosis. A close relationship was found between the visual colour scores and oxygen production (catalase activity) in Chaenomeles, but not in Chamaecyparis. The results agree with tests using gel chromatography as a means of determining the effectiveness of the chelates. Characterization of commercial iron chelates by this method before marketing is deemed necessary.
\end{abstract}

PEAT is widely used as a growing medium, having good aeration, moisture retention, and chemical buffering capacity. It contains few soil-borne diseases and weeds and is light in weight. Moss peat requires liming and the addition of nutrients to support plant growth.

The object of the present work was to test the effectiveness of some iron chelates to control $\mathrm{Fe}$ deficiency in ornamental shrubs grown on young sphagnum peat. The possibility of using gel chromatography (Boxma, 1979) to determine the effectiveness of the chelates was also examined.

\section{Experiment 1:}

$$
\text { MATERIALS AND METHODS }
$$

Chamaecyparis lawsoniana 'Alumii'

This cultivar was grown in a glasshouse with a sliding roof, starting in 1977. Each pot contained two plants which were harvested in October 1978. The 5-1 polyethylene pots contained $480 \mathrm{~g}$ of dry, slightly decomposed young sphagnum

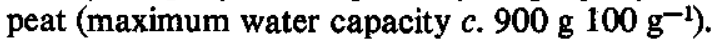
All pots received adequate amounts of $\mathrm{N}, \mathrm{P}, \mathrm{K}$, $\mathrm{Mg}, \mathrm{Cu}, \mathrm{B}, \mathrm{Mo}, \mathrm{Zn}$ and $\mathrm{Mn}$ as reagent grade chemicals. The treatments ( 3 replicates) included $0,1.56,3.12$ and $6.24 \mathrm{mg}$ Fe per pot as either $\mathrm{Fe}$ -
EDDHA (impure), Fe-EDTA or Fe-DTPA*, with 18,30 or $42 \mathrm{~g} \mathrm{CaCO}_{3}$ per pot. Supplemental dressings of iron chelates at half the above rates were added at the end of September 1977 and again in March 1978. Supplemental basic dressings of the essential nutrients $\mathrm{P}, \mathrm{Cu}, \mathrm{B}, \mathrm{Mo}$, $\mathrm{Zn}$ and $\mathrm{Mn}$ were added as a liquid feed at the start of the second year. A liquid feed supplying $0.51 \mathrm{~g} \mathrm{NH}_{4} \mathrm{NO}_{3}, 0.17 \mathrm{~g} \mathrm{~K}_{2} \mathrm{HPO}_{4}$ and $0.17 \mathrm{~g}$ $\mathrm{K}_{2} \mathrm{SO}_{4}$ per pot was also given fortnightly. The pots were watered daily with demineralized water to $c .80 \%$ of the maximum water retention. Dry weights (leaves + stems) and the visual colour scores (Fe deficiency) were analysed statistically (Duncan's multiple range and F-tests). The results from the gel chromatography test (Boxma, 1979) showed only one third of the water-soluble $\mathrm{Fe}(5.8 \%)$ in Fe-EDDHA (impure) to be present as Fe-EDDHA. In the other chelates, watersoluble $\mathrm{Fe}$ was largely present as Fe-EDTA (13.2\%) and Fe-DTPA (6.2\%), respectively. \footnotetext{
*Fe-EDDHA = ethylenediamine di (o-hydroxyphenylacetic

Fe-EDTA = ethylenediamine tetraacetic acid

Fe-DTPA $=$ diethylenetriamine pentaacetic acid
} 


\section{Experiment 2:}

Chaenomeles superba 'Nicoline'

This cultivar was used in this glasshouse experiment. Two rooted cuttings were planted in May and harvested in November 1978. The 5-1 polyethylene pots contained $415 \mathrm{~g}$ of dry, slightly decomposed young sphagnum peat (maximum water capacity $c .1000 \mathrm{~g}^{100 \mathrm{~g}^{-1}}$ ). All pots received adequate amounts of $\mathrm{N}, \mathrm{P}, \mathrm{K}, \mathrm{Mg}, \mathrm{Cu}$, $\mathrm{B}, \mathrm{Mo}, \mathrm{Zn}$ and $\mathrm{Mn}$ as reagent grade chemicals. The variable was $\mathrm{Fe}$ applied at rates of $0,1.0,2.5$, $5.0,7.5$ and $10.0 \mathrm{mg}$ per pot as either $\mathrm{Fe}$ EDDHA (pure, $6.4 \%$ ) or Fe-EDDHA (impure as used in Experiment 1). The chelates Fe-EDTA and Fe-DTPA (as used in Experiment 1) were added at rates of $2.5,5.0$ and $10.0 \mathrm{mg} F$ per pot. A solution of the impure Fe-EDDHA was eluted through a column of Sephadex G-10 $(1=100 \mathrm{~cm}$; diam. $=5 \mathrm{~cm}$ ), using deionized water as the eluent. This resulted in two coloured fractions. The first fraction leaving the column contained organic Fe compounds of moderate stability with a high molecular weight. The second fraction was the very stable $\mathrm{Fe}$ chelate Fe-EDDHA. Each fraction (effective $1.9 \%$ and ineffective $3.8 \% \mathrm{Fe}$ ) was added to the substrate at a rate of $5.0 \mathrm{mg} \mathrm{Fe}$ per pot. Treatments with $\mathrm{CaCO}_{3}$ were similar to those in Experiment 1. A liquid feed supplying $1.02 \mathrm{~g} \mathrm{NH}_{4} \mathrm{NO}_{3}, 0.34 \mathrm{~g} \mathrm{~K}_{2} \mathrm{HPO}_{4}$ and $0.34 \mathrm{~g}$ $\mathrm{K}_{2} \mathrm{SO}_{4}$ per pot was given fortnightly. Pots were watered daily with demineralized water to $c .80 \%$ of maximum water retention. The dry weights (leaves + stems) and the visual colour scores ( $\mathrm{Fe}$ deficiency) were analysed statistically (see Experiment 1).

\section{Analytical methods}

After ending the experiments, substrate samples were taken to determine $\mathrm{pH}-\mathrm{H}_{2} \mathrm{O}$. Prior to harvesting, top-leaves were sampled in some treatments to determine catalase activity so as to obtain an additional indication of the degree of $\mathrm{Fe}$ deficiency (Meakly and Chance, 1954).

\section{RESULTS}

Experiment 1: Chamaecyparis lawsoniana 'Alumii'

In the first year (1977) there was a slight general chlorosis, which was more severe in plants having little or no $\mathrm{Fe}$ at the high $\mathrm{pH}$. The impure chelate Fe-EDDHA proved inferior, $\mathrm{Fe}$ deficiency symptoms occurring in all treatments more or less irrespective of $\mathrm{pH}$.

Figure 1 illustrates the effects of iron chelates on chlorosis rating in 1978. The trends were basically similar to those in 1977 , but no visual $\mathrm{Fe}$ deficiency occurred at the lowest lime level $(\mathrm{pH}$ 3.6). The highest level of Fe-EDDHA (impure) was still sub-optimal at $\mathrm{pH} 4.7$ and 5.6. Chlorosis intensity was markedly lower for Fe-EDTA and Fe-DTPA, the difference being significant at $P=0.01$ for all corresponding amounts of iron.

\section{Catalase activity}

Prior to harvesting, leaf samples were taken from the high-lime treatments and analysed for

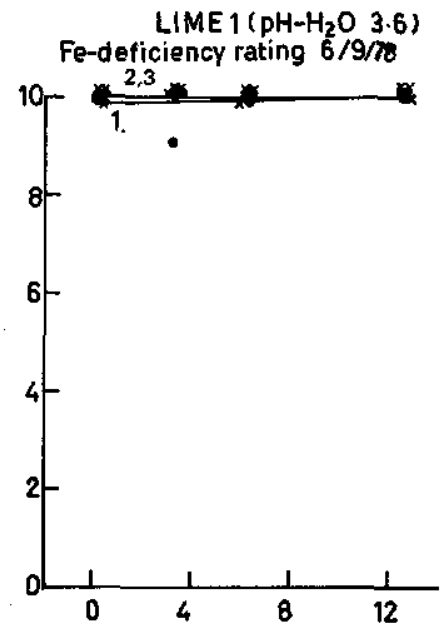

LIME $2\left(\mathrm{pH}-\mathrm{H}_{2} \mathrm{O} \quad 4 \cdot 7\right)$

LIME $3\left(\mathrm{pH}-\mathrm{H}_{2} \mathrm{O} 5.6\right)$
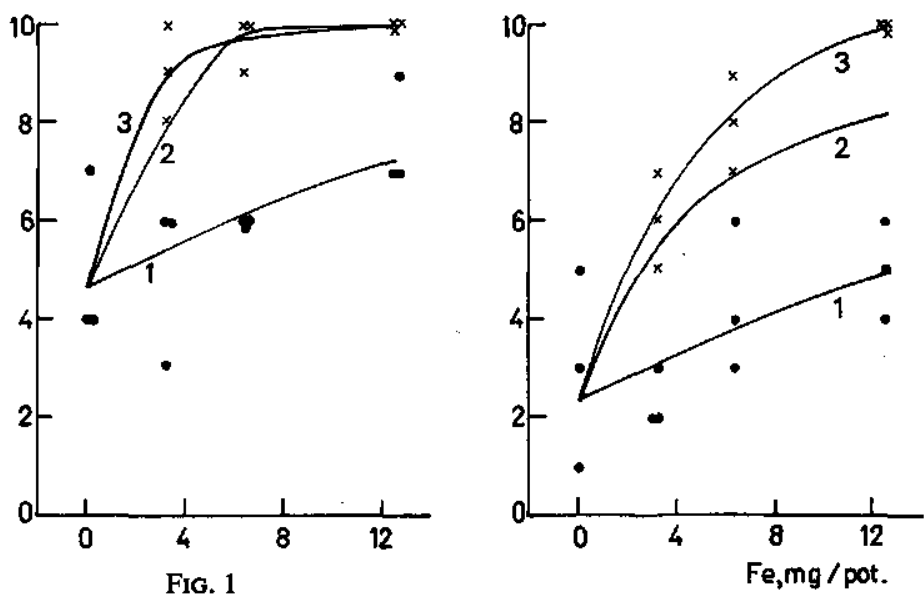

Chlorosis rating (iron deficiency) of Chamaecyparis on moss peat as affected by added iron chelates and $\mathrm{pH}$. Visual chlorosis score: $10=$ dark green and $1=$ yellow. Datum points: $\bullet=$ Fe-EDDHA (impure) $x=$ Fe-DTPA. $1=$ Fe-EDDHA (impure); 2 = Fe-EDTA; 3 = Fe-DTPA 
catalase activity. Nor relationship was found between catalase activity and $\mathrm{Fe}$ deficiency (chlorosis) rating.

\section{Crop yield}

The curves for chlorosis rating (Figure 1) and dry-matter yield (Figure 2, stem + leaves) are in close agreement. Figure 2 illustrates the inferior effect of Fe-EDDHA (impure) on the dry-matter yield of Chamaecyparis. Compared with $\mathrm{Fe}$ EDDHA, the yield was markedly higher for FeEDTA and Fe-DTPA; at pH 3.6, the difference was significant at $P=0.05$ only for the lowest amount of $\mathrm{Fe}$. At $\mathrm{pH} 4.7$ the differences for all three corresponding levels of $\mathrm{Fe}$ were significant. Possibly due to growth inhibition at $\mathrm{pH} 5.6$, the difference between Fe-DTPA and the other chelates was found to be significant only at the highest level of $\mathrm{Fe}$ at that $\mathrm{pH}$.

\section{Experiment 2: Chaenomeles superba 'Nicoline'} Following the results from Experiment 1, a further study was deemed necessary to compare the effectiveness of the chelates using pure $\mathrm{Fe}$ EDDHA as a standard.

\section{Observations during growth}

The effect of added $\mathrm{Fe}$ chelates on chlorosis rating (Fe deficiency) is illustrated in Figure 3.
The low-grade Fe-EDDHA, containing only one third of its $\mathrm{Fe}$ as Fe-EDDHA, proved ineffective as shown by chlorosis intensity and the earliness of its appearance. However, there was a large difference between the Fe-EDDHA fraction isolated from the low-grade product, which was almost as effective as pure Fe-EDDHA and the remaining fraction. At $\mathrm{pH} 5.6$ and above pure $\mathrm{Fe}$ EDDHA was a more effective $\mathrm{Fe}$ source than either Fe-EDTA or Fe-DTPA. At pH 4.5 there was only slight chlorosis in Chaenomeles and differences in effectiveness between chelates could not be assessed. Differences in chlorosis rating, at all corresponding amounts of $\mathrm{Fe}$, between pure and low-grade Fe-EDDHA were found to be significant $(P=0.05)$ at $\mathrm{pH} 5.6$ and 6.3 . At all pH levels $(4.5,5.6$ and 6.3$)$ differences in chlorosis rating between the effective and ineffective fraction of Fe-EDDHA (impure) were significant at $P=0.05$.

\section{Catalase activity}

The effects of $\mathrm{Fe}$ chelates on oxygen production (catalase activity) (Figure 4, left) are in agreement with the results illustrated in Figure 3 for chlorosis rating ( $\mathrm{pH}$ 6.3). Figure 4 (right) presents the close relationship between the visual colour scores and oxygen production. These graphs are in contrast with the results obtained with Chamaecyparis (Experiment 1).
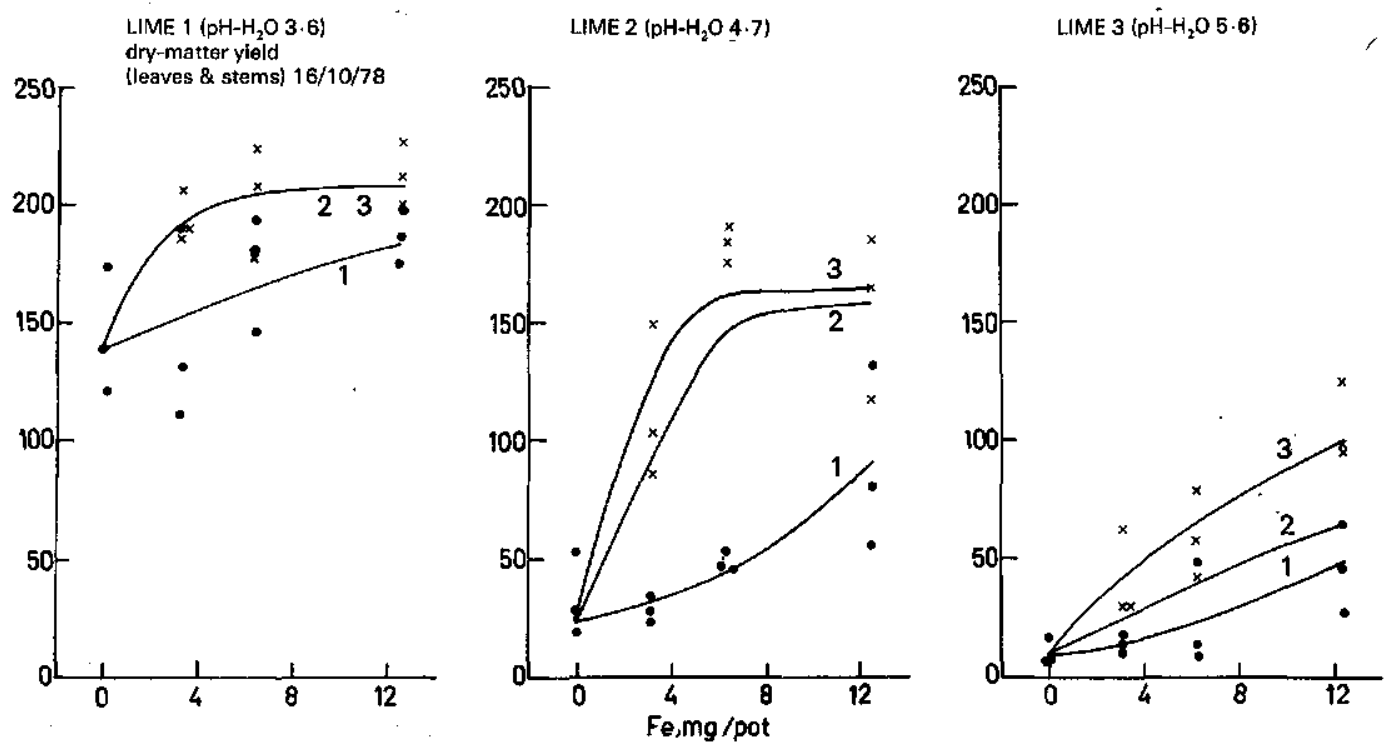

FIG. 2

Dry-matter yields of Chamaecyparis on moss peat as affected by added iron chelates and pH. Datum points: $\bullet=$ Fe-EDDHA (impure) $\mathrm{x}=\mathrm{Fe}-\mathrm{DTPA}$. 

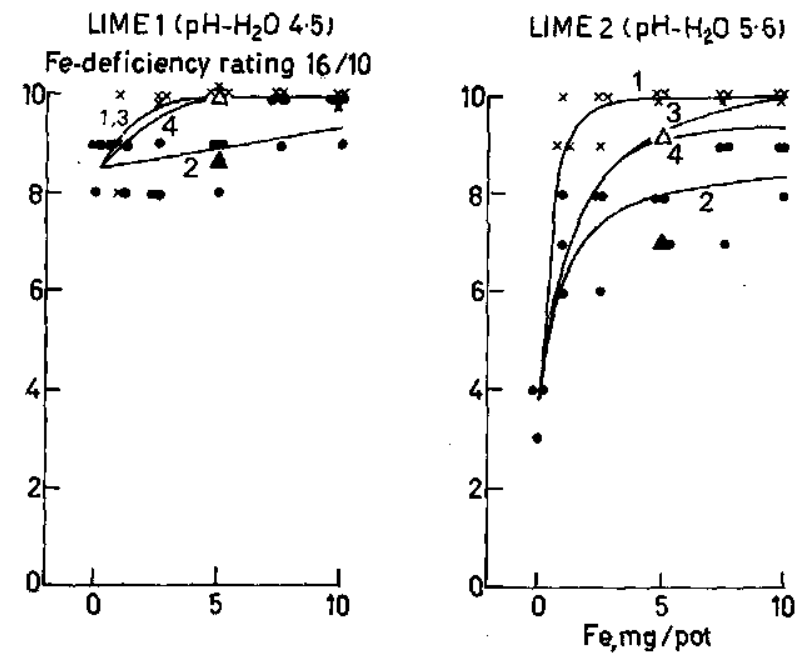

LIME $3\left(\mathrm{pH}-\mathrm{H}_{2} \mathrm{O} 6.3\right)$

FIG. 3

Chlorosis rating (iron deficiency) of Chaenomeles on moss peat as affected by added iron chelates and $\mathrm{pH}$. Visual chlorosis score $10=$ dark green and $\mathrm{I}=$ yellow. Datum points: $\bullet=$ Fe-EDDHA (impure) $\mathrm{x}=\mathrm{Fe}-\mathrm{EDDHA}$ (pure)

\section{LIME $3\left(\mathrm{pH}-\mathrm{H}_{2} \mathrm{O} 6.3\right)$}
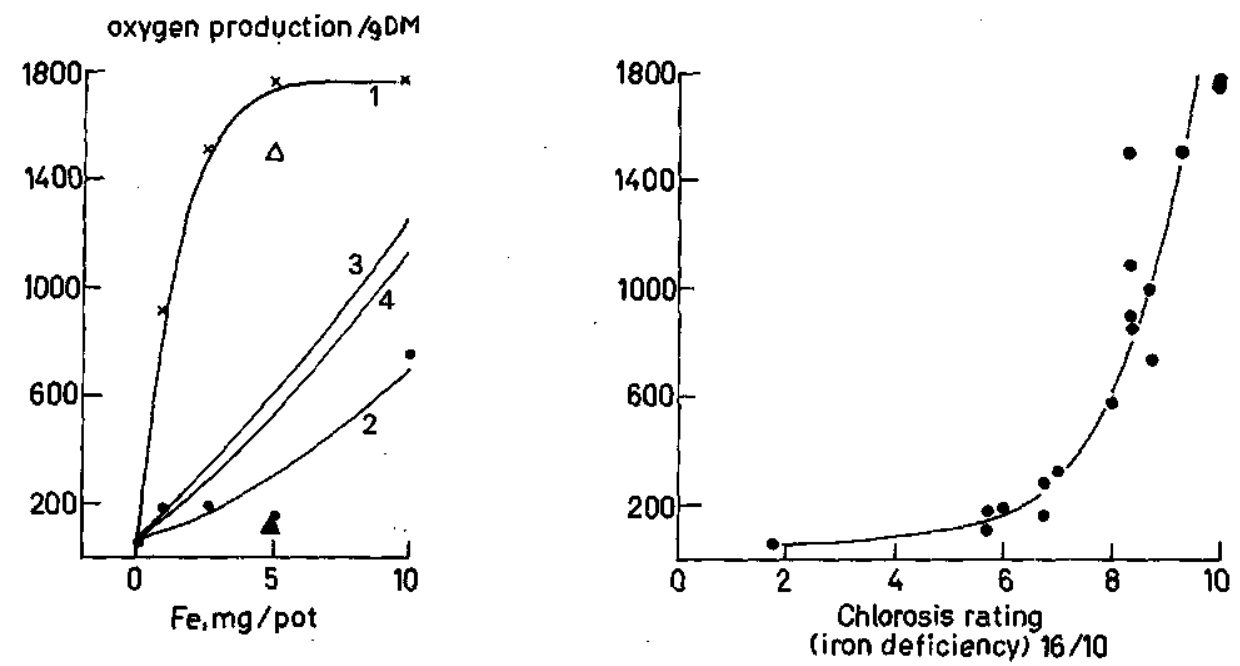

FIG. 4

Effect of iron chelates in moss peat on the catalase activity (left) and the relationship between chlorosis rating and the catalase activity (right) of Chaenomeles at $\mathrm{pH}$ 6.3. Datum points (left) $\bullet=\mathrm{Fe}-\mathrm{EDDHA}$ (impure) $\mathrm{x}=\mathrm{Fe}-\mathrm{EDDHA}$ (pure)

$1=$ Fe-EDDHA (pure); $2=$ Fe-EDDHA (impure); $3=$ Fe-EDTA; $4=$ Fe-DTPA; $\Delta=$ Effective fraction Fe-EDDHA (impure product); $A=$ Ineffective fraction Fe-EDDHA (impure product)

\section{Crop yield}

Chlorosis ratings (Figure 3 ) are in close agreement with the dry-matter yields of Chaenomeles. It is noteworthy that Fe-DTPA is less effective than (pure) Fe-EDDHA in controlling $\mathrm{Fe}$ deficiency at $\mathrm{pH} 5.6$ and 6.3. The best overall results were obtained with Fe-EDDHA (pure), which also proved superior to Fe-EDTA at $\mathrm{pH}>5.6$.
The results obtained with low-grade Fe-EDDHA were inferior at all $\mathrm{pH}$ levels. Differences among Fe sources were highly significant, but, because of uneven growth, not at all corresponding amounts of $\mathrm{Fe}(\mathrm{S} \%=30.6)$.

DISCUSSION

To date, little attention has been given to devis- 
ing analyses to check the quality of commercial Fe chelates. Various chromatographic methods, such as paper and TLC, ion-exchange and gas chromatography (Hill-Cottingham, 1957; Rajabalee et al., 1973; Longbottom, 1972; Aue et al., 1972) have been used for this purpose. Almost all suffer from cation interference, lack of sensitivity or inability to determine more than one or two chelates.

The gel chromatographic analysis (Boxma, 1979), based on a separation technique, proved suitable for the determination of individual $\mathrm{Fe}$ chelates. It showed that in a commercial $\mathrm{Fe}$ -
EDDHA chelate only one third of the total soluble $\mathrm{Fe}$ was present as pure Fe-EDDHA. This agrees with the lack of effectiveness of this chelate in controlling $\mathrm{Fe}$ deficiency in some indicator plants.

In the present work the best overall results were obtained with pure Fe-EDDHA, which was a more effective $\mathrm{Fe}$ source than either Fe-EDTA or Fe-DTPA at high pH levels. These conclusions are similar to those of Wallace et al. (1955), Holmes and Brown (1955) and Kuykendall et al. (1957) for calcareous soils.

\section{REFERENCES}

Aue, W. A., Hastings, C. R., Gerhardt, K., Pierce, J. O., Hill, H. H. and Moseman, R. F. (1972). The determination of part-per billion levels of citric and nitrilotriacetic acid in tapwater and sewage effluents. Journal of Chromatography, 72, 259-67.

BoxмA, R. (1979). Analysis of iron chelates in commercial iron fertilizers by gel chromatography. Zeitschrift für Pflanzenernährung und Bodenkunde, 142, 824-35.

HILl-Cortingham, D. G. (1957). A spectrophotometric method of analysis of chelate solutions and its application to the study of iron chelates in soils and plants. Soll Science, 84, 43-9.

Holmes, R. S. and BRown, J. C. (1955). Chelates as correctives for chlorosis. Soil Science, 80, 167-79.

KuYkendall, J. R., Hilgeman, R. H. and VAN HoRN, C. W. (1957). Response of chlorotic citrus trees in Arizona to soil applications of iron chelates. Soil Science, 84, 77-86.

LoNGBotTom, J. E. (1972). Determination of nitrilotriacetic acid by high-speed ion exchange chromatography. Analytical Chemistry, 44, 418-20.

MAEkLY, A. C. and ChANCE, B. (1954). Methods of biochemical analysis. Interscience, 1, New York, 357.

Rajabalee, T. J. M., Potvin, M. and Laham, S. (1973). Separation of NTA and EDTA chelates by thin-layer chromatography. Journal of Chromatography, 79, 375-9.

Wallace, A., Mueller, R. T., Lunt, D. R., Ashcroft, R. T. and Shannon, L. M. (1955). Comparisons of five chelating agents in soils, in nutrient solutions and in plant responses. Soil Science, 80, 101-8.

(Accepted 28 October 1980) 